\title{
Editorial
}

\section{Exotic Superconductivity in Correlated Electron Systems}

\author{
Gang Mu, ${ }^{1}$ Viorel Sandu, ${ }^{2}$ Wei Li, ${ }^{1}$ and Bing Shen ${ }^{3}$ \\ ${ }^{1}$ State Key Laboratory of Functional Materials for Informatics and Shanghai Center for Superconductivity, \\ Shanghai Institute of Microsystem and Information Technology, Chinese Academy of Sciences, Shanghai 200050, China \\ ${ }^{2}$ National Institute of Materials Physics, 077125 Măgurele, Romania \\ ${ }^{3}$ Argonne National Laboratory, Argonne, IL 60439, USA
}

Correspondence should be addressed to Gang Mu; mugang@mail.sim.ac.cn

Received 25 May 2015; Accepted 25 May 2015

Copyright ( $\odot 2015$ Gang Mu et al. This is an open access article distributed under the Creative Commons Attribution License, which permits unrestricted use, distribution, and reproduction in any medium, provided the original work is properly cited.

Over the past decades, the search for high- $\mathrm{T}_{\mathrm{c}}$ superconductivity (SC) and its novel superconducting mechanisms is one of the most challenging tasks of condensed matter physicists and material scientists, wherein the most striking achievement is the discovery of high- $\mathrm{T}_{\mathrm{c}}$ and unconventional superconductivity in strongly correlated 3d-electron systems, such as cuprates and iron pnictides/chalcogenides. Those exotic superconductors display the behaviors beyond the scope of the BCS theory (in the SC states) and the LandauFermi liquid theory (in the normal states). In general, such exotic superconductivity can be seen as correlated electron systems, where there are strong interplays among charge, spin, orbital, and lattice degrees of freedom. Thus, we focus on the exotic superconductivity in materials with correlated electrons in the present special issue.

To address the fruitful aspects in this special issue, we invited the authors ranging from experimentalists to theorists to submit their original researching papers. The topics also range from material synthetizing to physical investigations. In the former topic, two papers made efforts to explore new superconducting materials in different systems through different approaches. In the latter topic, one paper investigated the crystal field excitations in an iron-pnictide superconductor using the inelastic neutron scattering. Another two papers focused on the gap structure of the unconventional cuprates and iron-pnictide superconductors.

The paper by B. Li et al. reported pressure induced suppression to the valence change transition in a $4 \mathrm{~d}$-electron compound EuPdAs. Two-phase transitions at about $10 \mathrm{~K}$ and $180 \mathrm{~K}$, respectively, were observed from resistivity and magnetic susceptibility measurements. They attempted to explore superconductivity in this system by applying pressure. They found that the transition at $180 \mathrm{~K}$ can be suppressed with a pressure as low as $0.48 \mathrm{GPa}$ and no superconductivity has been induced with the pressure up to $1.90 \mathrm{GPa}$. Y. B. Sun et al. reported the insulator to superconductor transition in Gadoped semiconductor Ge single crystal. The rapid thermal annealing was found to make Ga redistribute in the Ge matrix and realize the superconducting circuits. With increasing the annealing temperature, the samples show a crossover from the insulator to the superconductor. They argued that the Ga doped layer plays a leading role in the superconductivity.

Iron-pnictide superconductor $\mathrm{NdFeAsO}_{0.85} \mathrm{~F}_{0.15}$ was investigated by $\mathrm{P}$. Cheng et al. using inelastic neutron scattering measurements. They used the polycrystalline samples and the experiments were carried out over a wide temperature range of $3 \mathrm{~K}-250 \mathrm{~K}$. They identified up to seven ground state crystal field excitations from the inelastic neutron spectra. A crystal field energy level scheme for $\mathrm{Nd}^{3+}$ was proposed. It shows quite different features comparing with that in tetragonal parent phase $\mathrm{NdFeAsO}$. Their results indicate that the fluorine doping induced the superposition of crystal fields and lowered local point symmetries around the $\mathrm{Nd}^{3+}$.

The symmetry and structure of the energy gap are one of the most important issues in the studies of the high$\mathrm{T}_{c}$ and unconventional superconductors. Recently it was revealed by angle-resolved photoemission spectroscopy that the whole Fermi surface is fully gapped for several families of underdoped cuprates, which challenges the present theories 
for the high- $\mathrm{T}_{\mathrm{c}}$ superconductors. Based on a phenomenological model, T. Zhou et al. propose that the incommensurate diagonal spin-density-wave order can cause a finite gap along the d-wave nodal line. Their work gives a good explanation to the experimental observation. G. Mu et al. studied the gap structure of the iron-pnictide superconductor $\mathrm{Ba}\left(\mathrm{Fe}_{0.942} \mathrm{Ni}_{0.058}\right)_{2} \mathrm{As}_{2}$ by the low-temperature specific heat measurements. A simple and reliable analysis shows that a clear $\mathrm{T}^{2}$ term emerges in the low-temperature specific heat data. Their observation is similar to that observed in the Co-doped system in their previous work and is consistent with the theoretical prediction for a superconductor with line nodes in the energy gap.

\section{Acknowledgments}

At last, we sincerely acknowledge all the contributors for their hard work and patience in bringing out this special issue. We hope that the publication of this special issue will be of reference value for readers.

Gang $M u$

Viorel Sandu

Wei $\mathrm{Li}$

Bing Shen 

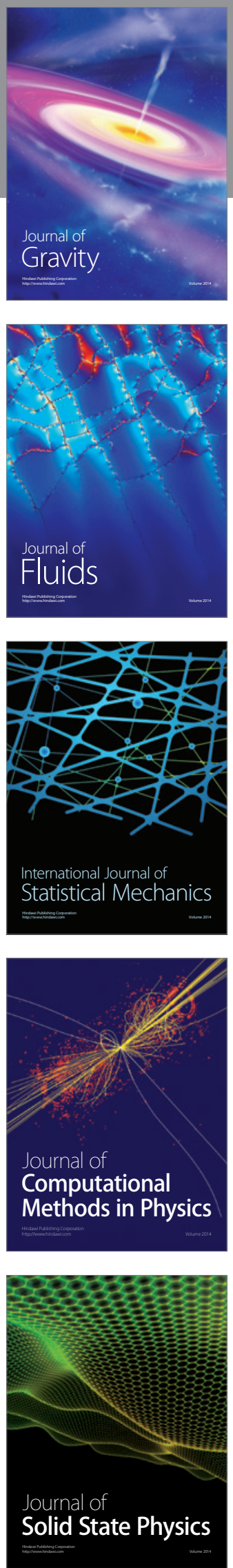

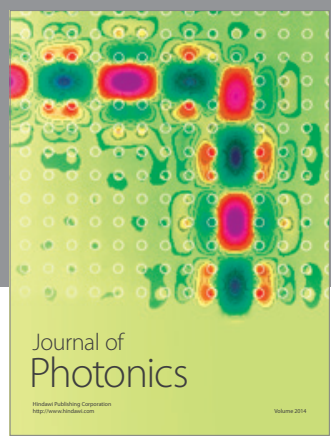

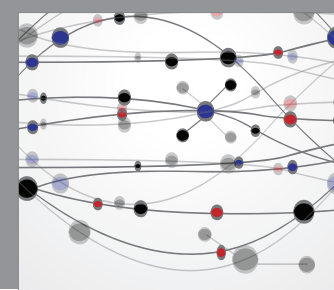

The Scientific World Journal

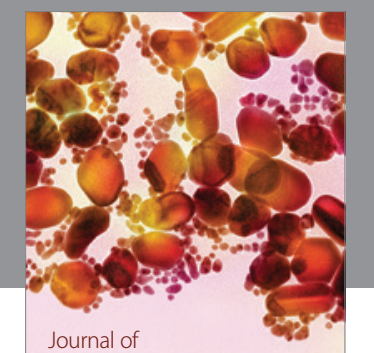

Soft Matter
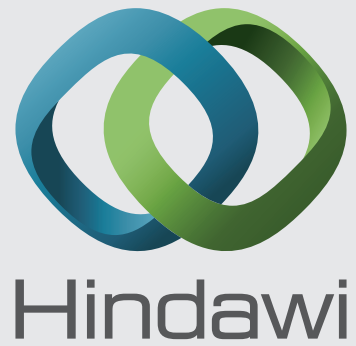

Submit your manuscripts at

http://www.hindawi.com
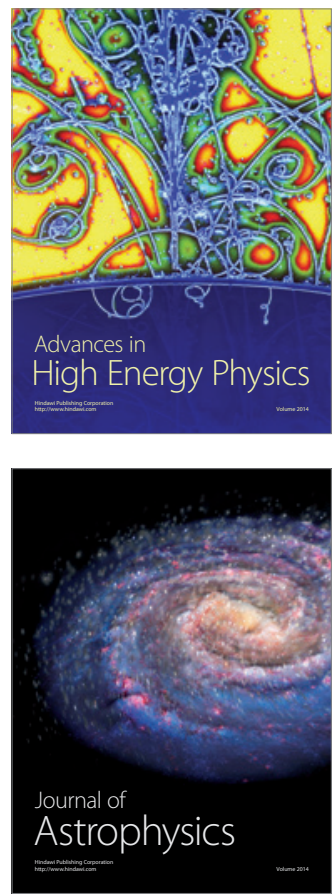
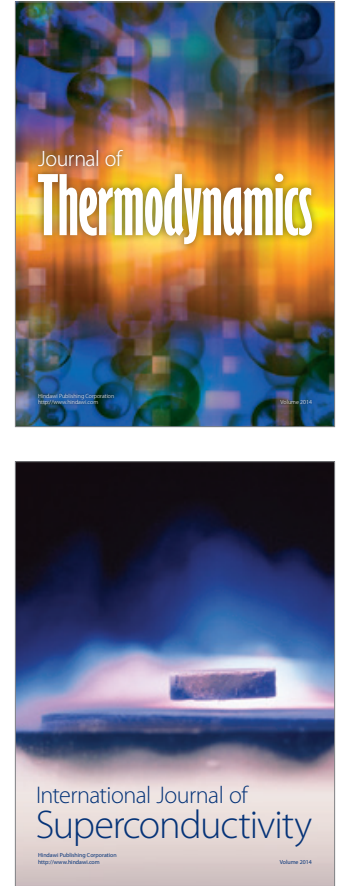
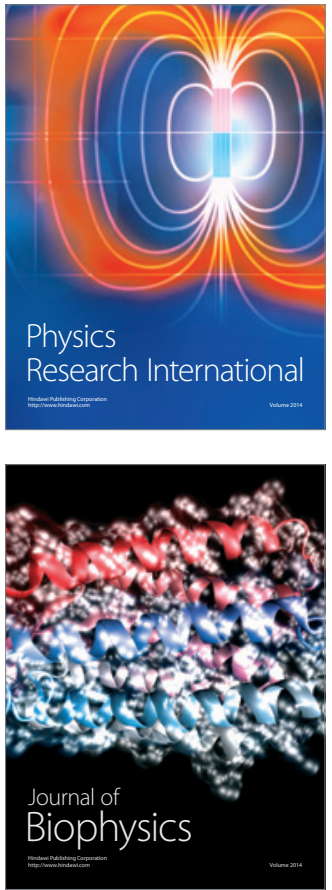
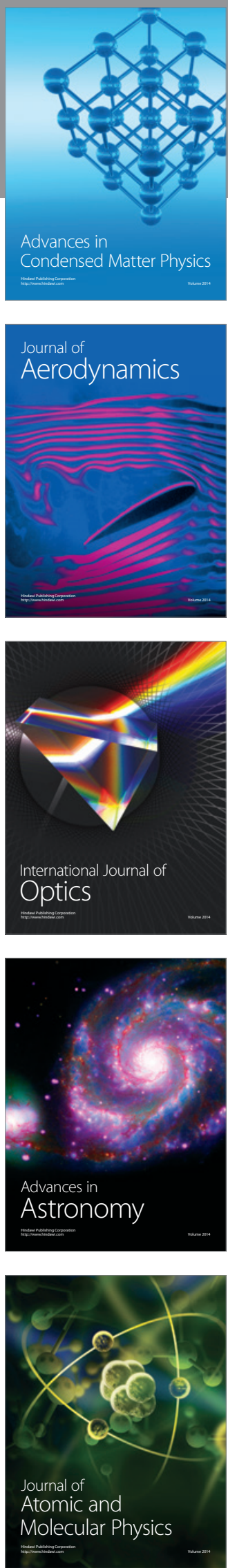